\title{
Supplementary material for: On a Dirichlet process mixture representation of phase-type distributions
}

\author{
Daniel Ayala * $\quad$ Leonardo Jofré ${ }^{\dagger} \quad$ Luis Gutiérrez $^{\ddagger} \quad$ Ramsés H. Mena ${ }^{\S}$
}

May 17, 2021

\section{Appendix}

\section{Posterior inference derivations}

For $\phi_{h}, h=1, \ldots, N$, we have that

$$
\begin{aligned}
p\left(\phi_{h} \mid \ldots\right) & \propto\left\{\prod_{\left\{k: d_{k}=h\right\}} \operatorname{Er}\left(y_{k} \mid\left\lceil\phi_{h}\right\rceil, \lambda\right)\right\} \times \pi\left(\phi_{h}\right) \\
& =\left\{\prod_{\left\{k: d_{k}=h\right\}} \frac{\lambda^{\left\lceil\phi_{h}\right\rceil} y_{k}^{\left\lceil\phi_{h}\right\rceil-1} e^{-\lambda y_{k}}}{\left(\left\lceil\phi_{h}\right\rceil-1\right) !}\right\} \times \frac{b_{0}^{a_{0}}}{\Gamma\left(a_{0}\right)} \phi_{h}^{a_{0}-1} e^{-b_{0} \phi_{h}} \\
& \propto\left\{\prod_{\left\{k: d_{k}=h\right\}} \frac{\lambda^{\left\lceil\phi_{h}\right\rceil} y_{k}^{\left\lceil\phi_{h}\right\rceil-1}}{\left(\left\lceil\phi_{h}\right\rceil-1\right) !}\right\} \times \phi_{h}^{a_{0}-1} e^{-b_{0} \phi_{h}}
\end{aligned}
$$

Note that there is no closed form for this posterior distribution. Consequently, we resort to the Metropolis-Hastings (M-H) algorithm (Metropolis et al. 1953 Hastings; 1970) to obtain samples from (1).

For the parameter $\lambda$, we assume a prior distribution $\operatorname{Gamma}\left(a_{1}, b_{1}\right)$ (see hierarchical model (8)).

* Departamento de Estadística, Pontificia Universidad Católica de Chile

†Departamento de Estadística, Pontificia Universidad Católica de Chile

${ }^{\ddagger}$ Departamento de Estadística, Pontificia Universidad Católica de Chile, and Millenium Nucleus Center for the Discovery of Structures in Complex Data

§IIMAS-UNAM, México 
Therefore, its posterior distribution can be expressed as:

$$
\begin{aligned}
p(\lambda \mid \ldots) & \propto \mathcal{L}(\boldsymbol{w}, \boldsymbol{\phi}, \lambda \mid \boldsymbol{y}, \boldsymbol{d}, \boldsymbol{u}) \pi(\lambda) \\
& =\left\{\prod_{k=1}^{n} \mathbb{1}\left\{u_{k}<w_{d_{k}}\right\} \operatorname{Er}\left(y_{k} \mid\left\lceil\phi_{d_{k}}\right\rceil, \lambda\right)\right\} \times \operatorname{Gamma}\left(a_{1}, b_{1}\right) \\
& \propto\left\{\prod_{k=1}^{n} \lambda^{\left\lceil\phi_{d_{k}}\right\rceil} e^{-\lambda y_{k}}\right\} \times \lambda^{a_{1}-1} e^{-b_{1} \lambda} \\
& \propto \lambda^{a_{1}+\sum_{k}\left\lceil\phi_{d_{k}}\right\rceil-1} e^{-\lambda\left(b_{1}+\sum_{k} y_{k}\right)} . \\
\therefore \quad(\lambda \mid \ldots) & \sim \operatorname{Gamma}\left(a_{1}+\sum_{k=1}^{n}\left\lceil\phi_{d_{k}}\right\rceil, b_{1}+\sum_{k=1}^{n} y_{k}\right) .
\end{aligned}
$$

The posterior distributions for $v_{h}, u_{k}$ and $d_{k}$ are given by:

$$
\begin{aligned}
\left(v_{h} \mid \ldots\right) & \sim \operatorname{Beta}\left(1+\sum_{k=1}^{n} \mathbb{1}\left\{d_{k}=h\right\}, \alpha+\sum_{k=1}^{n} \mathbb{1}\left\{d_{k}>h\right\}\right), \\
\left(u_{k} \mid \ldots\right) & \sim \operatorname{Unif}\left(0, w_{d_{k}}\right) \\
\mathbb{P}\left[d_{k}=h \mid \ldots\right] & \propto \mathbb{1}\left\{h: w_{h}>u_{k}\right\} \operatorname{Er}\left(y_{k} \mid\left\lceil\phi_{d_{k}}\right\rceil, \lambda\right)
\end{aligned}
$$

as shown in Kalli et al. (2011), which can be easily sampled from.

By letting a prior density $\alpha \sim \operatorname{Gamma}\left(a_{\alpha}, b_{\alpha}\right)$, the resulting posterior distribution is of the form (Escobar and West, 1995):

$$
\begin{aligned}
(\alpha \mid \eta, N) \sim \omega_{\eta} & \operatorname{Gamma}\left(a_{\alpha}+N, b_{\alpha}-\log (\eta)\right) \\
+\left(1-\omega_{\eta}\right) \operatorname{Gamma}\left(a_{\alpha}+N-1, b_{\alpha}-\log (\eta)\right) &
\end{aligned}
$$

where the mixture weight $\omega_{\eta}$ satisfies

$$
\frac{\omega_{\eta}}{\left(1-\omega_{\eta}\right)}=\frac{\left(a_{\alpha}+N-1\right)}{n\left(b_{\alpha}-\log (\eta)\right)}
$$

and $\eta$ is a random quantity with posterior distribution

$$
(\eta \mid \alpha, N) \sim \operatorname{Beta}(\alpha+1, n)
$$


Monte Carlo results for sample sizes 125 and 250
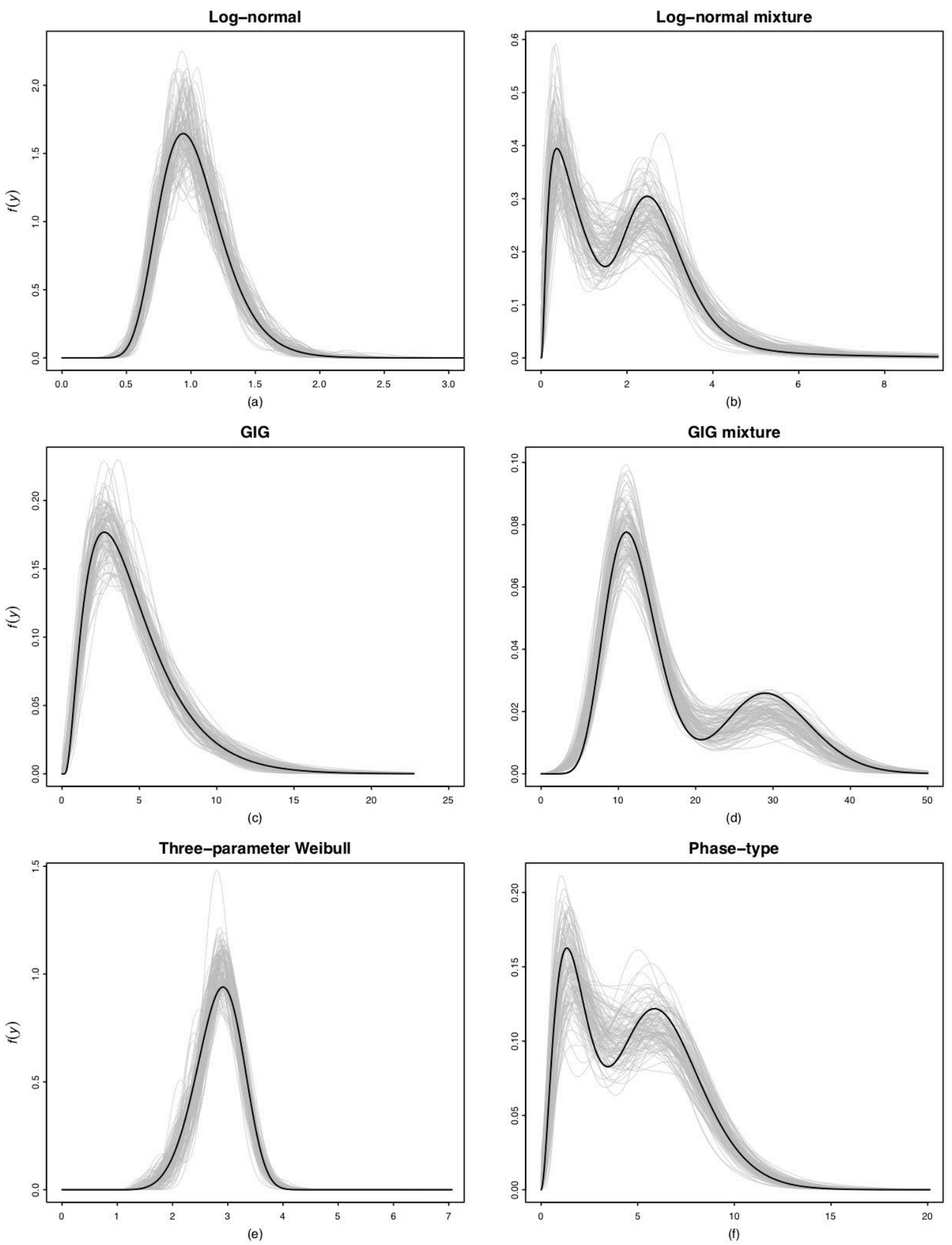

Figure 1: True density function (solid lines) and posterior mean density estimates (dotted lines) of the 100 Monte Carlo runs for the selected distributions and sample size $n=125$ 

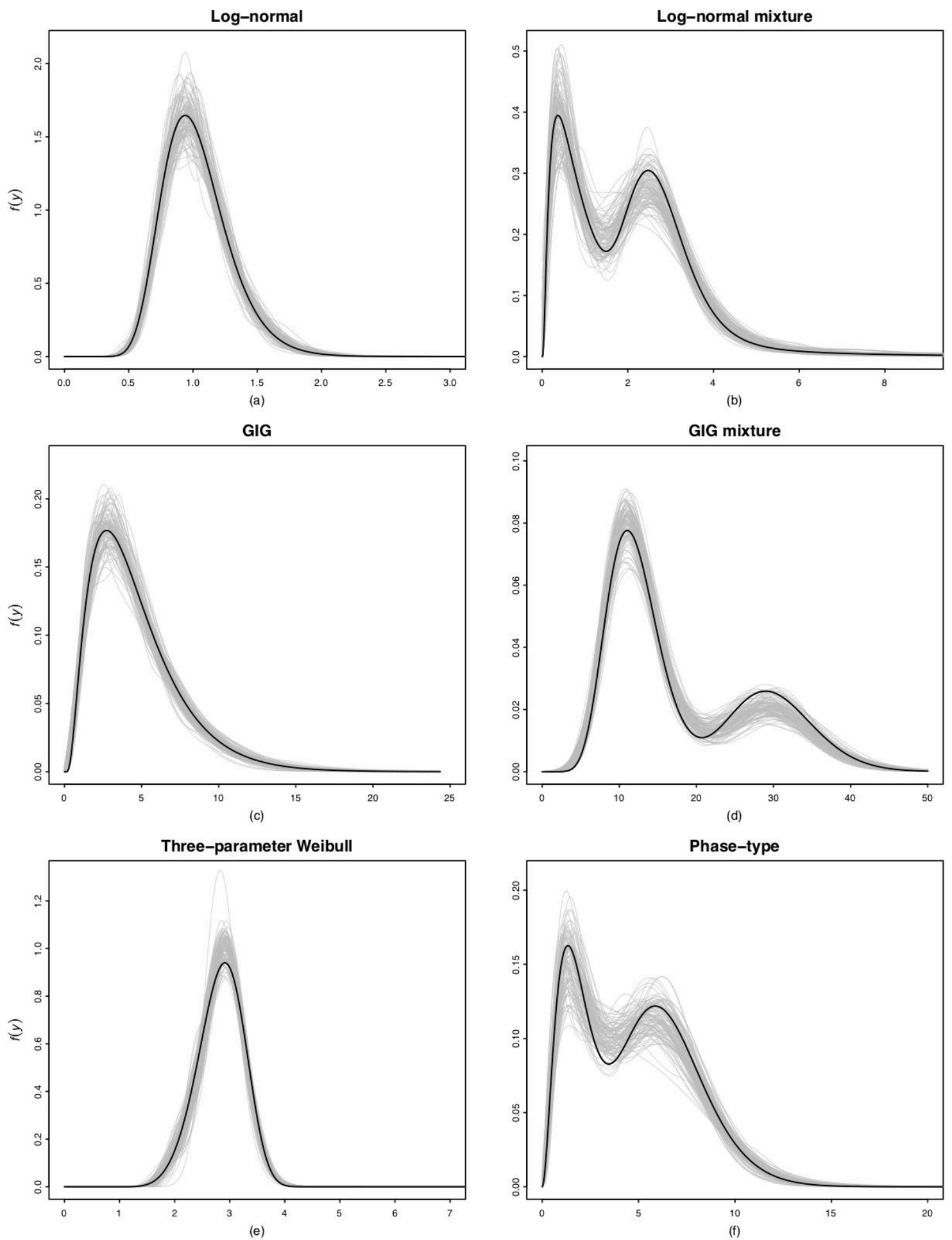

Figure 2: True density function (solid lines) and posterior mean density estimates (dotted lines) of the 100 Monte Carlo runs for the selected distributions and sample size $n=250$ 


\section{$\mathbf{R}$ function for posterior inference}

The function mcmcErlangMix produces posterior samples from model (8). The arguments of the function are:

Y: data as a vector,

a: hyperparameter $a_{1}$,

b: hyperparameter $a_{2}$,

aa: hyperparameter $a_{0}$,

$\mathrm{bb}$ : hyperparameter $b_{0}$,

alpha: hyperparameter $a_{\alpha}$,

beta: hyperparameter $b_{\alpha}$,

nscan: number of iterations,

nburn: burn-in period,

nskip: thinning value.

The result of the function is a matrix with posterior realizations of the density. The following example reproduces a realization for the Generalized Inverse Gaussian mixture of Table 1.

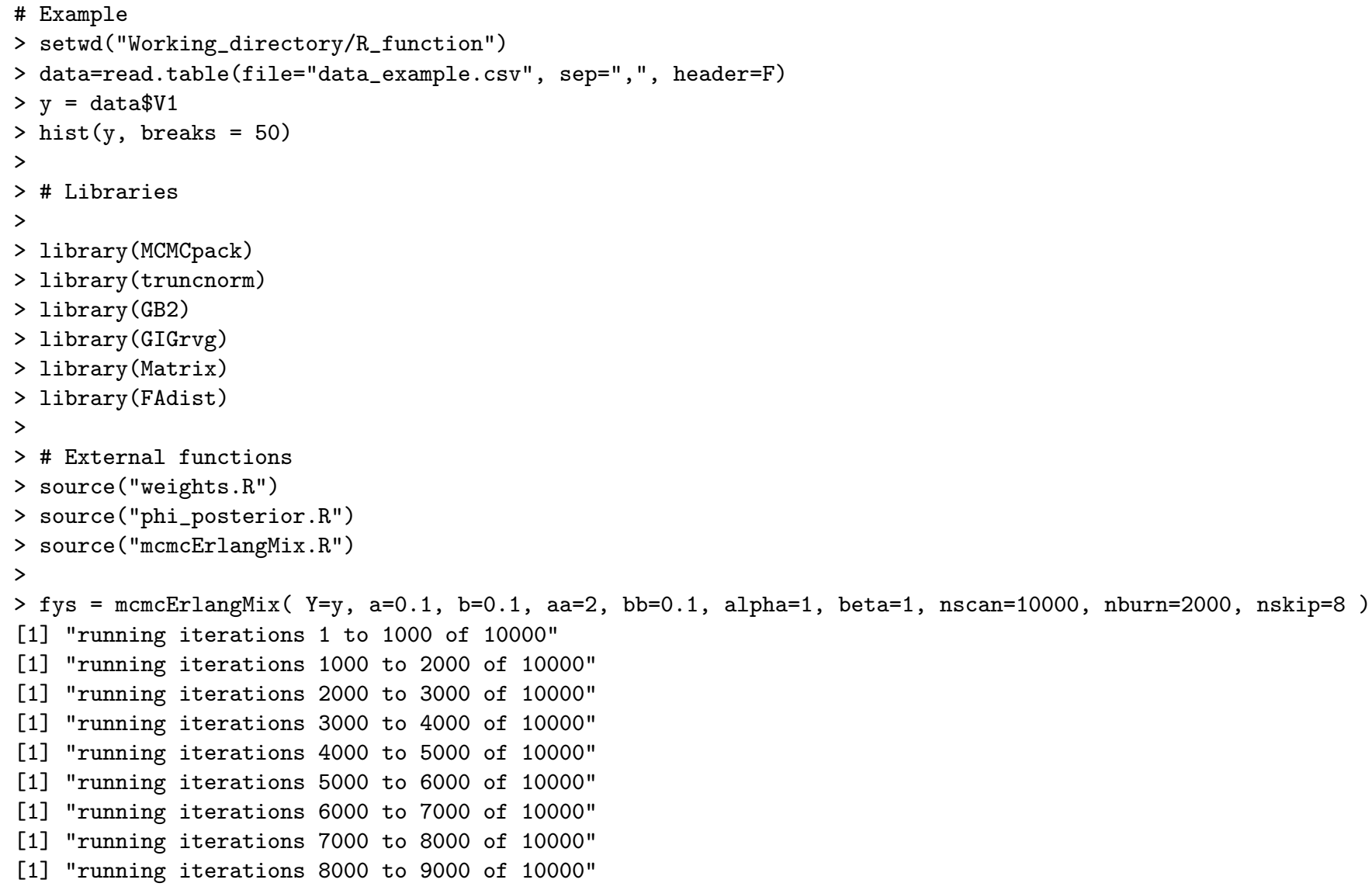



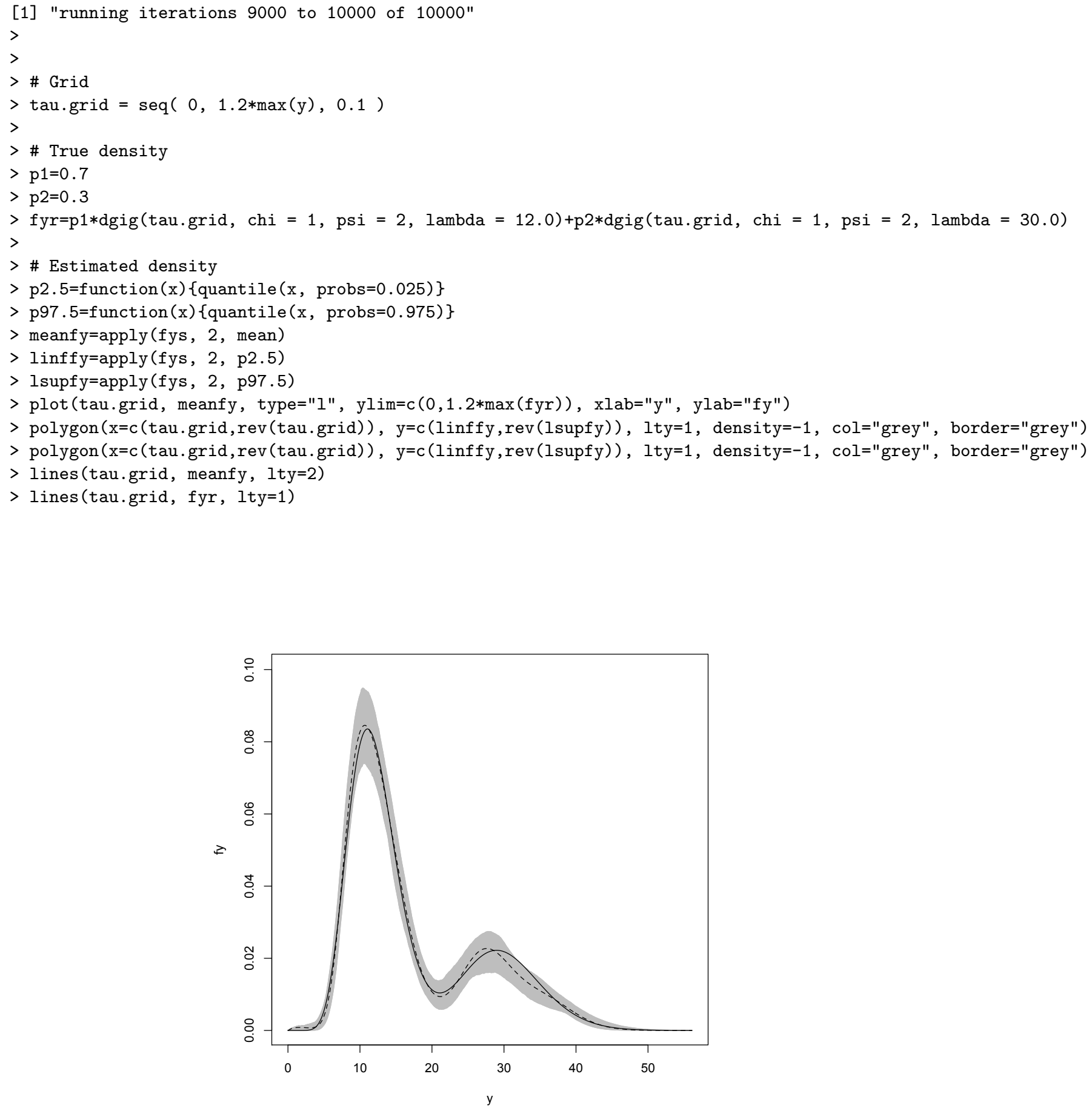

Figure 3: Posterior mean estimates (solid lines) and 95\% credible intervals (shaded areas) for a realization of a GIG mixture. 


\section{References}

Escobar, M. D. and West, M. (1995). Bayesian density estimation and inference using mixtures, Journal of the American Statistical Association 90(430): 577-588.

Hastings, W. K. (1970). Monte carlo sampling methods using markov chains and their applications, Biometrika 57(1): 97-109.

Kalli, M., Griffin, J. E. and Walker, S. G. (2011). Slice sampling mixture models, Statistics and Computing 21(1): 93-105.

Metropolis, N., Rosenbluth, A. W., Rosenbluth, M. N., Teller, A. H. and Teller, E. (1953). Equation of state calculations by fast computing machines, The Journal of Chemical Physics 21(6): 1087-1092. 